\title{
Hesperidin protects against IL-1 $\beta$-induced inflammation in human osteoarthritis chondrocytes
}

\author{
ZHAOZONG FU, ZHONGXIAN CHEN, QINGHUA XIE, HONGJUN LEI and SHANSHAN XIANG
}

Department of Spine Surgery, Jiangmen Central Hospital, Jiangmen, Guangdong 529030, P.R. China

Received February 7, 2018; Accepted May 25, 2018

DOI: $10.3892 /$ etm.2018.6616

\begin{abstract}
Hesperidin is a vitamin P flavonoid compound primarily present in citrus fruits, which possesses an anti-inflammatory effect. The functional role of hesperidin in interleukin (IL)-1 $\beta$-stimulated human osteoarthritis (OA) chondrocytes is still unknown. In the present study, anti-inflammatory effects of hesperidin in IL-1 $\beta$-stimulated chondrocytes were investigated. The results demonstrated that hesperidin treatment markedly decreased nitric oxide and prostaglandin E2 production and markedly downregulated inducible nitric oxide synthase and cyclooxygenase- 2 expression in IL-1 $\beta$-stimulated OA chondrocytes. In addition, hesperidin markedly reduced IL-1 $\beta$-induced matrix metalloproteinase (MMP)-3 and MMP-13 expression in human OA chondrocytes. Furthermore, hesperidin markedly decreased the activation of nuclear factor (NF)- $\kappa \mathrm{B}$ in human OA chondrocytes. In conclusion, it was revealed for the first time that hesperidin inhibited inflammatory responses in IL-1 $\beta$-stimulated human chondrocytes, potentially through inhibiting the activation of the $N F-\kappa B$ signaling pathway. These data suggest that hesperidin may be a potential agent for the treatment of OA.
\end{abstract}

\section{Introduction}

Osteoarthritis (OA) is a degenerative disease, with patients exhibiting joint pain, joint swelling, restricted movement and joint deformities (1). OA is an autoimmune disease and an increasing number of patients $(\sim 8 \%)$ with OA have been diagnosed in the clinic since 2008 worldwide (2-4). OA is divided into primary and secondary OA, according to the presence of local and systemic risk factors, including inflammation and genetic factors (5). The causes of OA are complex, including genetic, physical and chemical factors, which are difficult to

Correspondence to: Professor Zhaozong Fu, Department of Spine Surgery, Jiangmen Central Hospital, 23 Haibang Street, Jiangmen, Guangdong 529030, P.R. China

E-mail: yalingyangprof@163.com

Key words: hesperidin, osteoarthritis, inflammation, nuclear factor $\kappa \mathrm{B}$ classify with regards to a systematic model (6). The pathogenesis associated with this disease is not well understood and OA is frequently misdiagnosed as rheumatoid arthritis or ankylosing spondylitis in clinical settings (7-9).

Hesperidin is a flavanone glycoside, which is a vitamin $\mathrm{P}$ flavonoid compound (10). A previous study reported biological activities of hesperidin and its molecular mechanisms, including involvement in the nuclear factor erythroid 2-related factor/extracellular signal-regulated kinase, No/cGFP and phosphoinositide 3-kinase/mitogen-activated protein kinase signaling pathways in neuropharmacology (11). In addition, the therapeutic effects of hesperidin on cardiac tissue and renal and hepatic damage have been evaluated in several reports (12-14). Another study revealed that hesperidin presented protective effects against radiation-induced hepatic injury and damage to peripheral blood lymphocytes (15). Furthermore, hesperidin ameliorates oxidative stress and mitochondrial dysfunction by activation of antioxidant enzymes, including superoxide dismutase, catalase and glutathione-S-transferase (16). In addition, hesperidin alleviates acetaminophen-induced toxicity in Wistar rats by abrogation of oxidative stress, apoptosis and inflammation (17). However, the anti-inflammatory effects of hesperidin on inflammation in human osteoarthritis chondrocytes remain unclear.

A previous study indicated that cyclooxygenase (COX)-2-selective inhibitors may be used for the treatment of rheumatoid arthritis (18). Matrix metalloproteinase (MMP) protein and activity levels are upregulated in synovial fluid from patients with joint injury, inflammatory arthritis and osteoarthritis compared to healthy controls (19). Notably, nuclear factor (NF) $-\kappa \mathrm{B}$ activation has been observed to increase in OA articular chondrocytes (20). In the current study, the efficacy of hesperidin in interleukin (IL)-1 $\beta$-stimulated human chondrocytes was investigated. The study further explored potential mechanisms mediated by hesperidin in human chondrocytes and reported an association between hesperidin and NF- $\mathrm{NB}$ signaling pathway activation in IL-1 $\beta$-stimulated human chondrocytes.

\section{Materials and methods}

Cell culturing and treatment. Human OA chondrocytes were obtained from the Department of Anatomy \& Neurobiology, Northeast Ohio Medical University (Ohio, USA) and cultured in RPMI 1640 medium (Thermo Fisher 
Scientific, Inc., Waltham, MA, USA) supplemented with $10 \%$ heat-inactivated fetal bovine serum (Thermo Fisher Scientific, Inc.) at $37^{\circ} \mathrm{C}$ in $5 \% \mathrm{CO}_{2}$. Cells were treated with IL-1 $\beta(2.0 \mathrm{mg} / \mathrm{ml}$, Sigma-Aldrich; Merck KGaA, Darmstadt, Germany) and/or hesperidin $(2.0 \mathrm{mg} / \mathrm{ml}), \mathrm{NF}-\kappa \mathrm{B}$ inhibitor (NF-kBIR; cat. no. LY-294.002; 2.0 mg/ml, Sigma-Aldrich; Merck KGaA, Darmstadt, Germany) or PBS (Sigma-Aldrich; Merck KGaA, Darmstadt, Germany) for $24 \mathrm{~h}$ at $37^{\circ} \mathrm{C}$. The groups are as follows: PBS, IL- $1 \beta, \mathrm{NF}-\kappa \mathrm{BIR}$, hesperidin and IL-1 $\beta+$ hesperidin.

Reverse transcription-quantitative polymerase chain reaction (RT-qPCR) analysis. Total RNA from human OA chondrocytes $\left(1 \times 10^{8}\right.$ cells) was extracted using an RNAeasy Mini kit (Qiagen Sciences, Inc., Gaithersburg, MD, USA) following the manufacturer's protocol. RNA was reverse transcribed into cDNA at $42^{\circ} \mathrm{C}$ for $2 \mathrm{~h}$ using the High Capacity cDNA Reverse Transcription kit (Thermo Fisher Scientific, Inc.) according to the manufacturer's protocol. Expression levels of mRNAs in cells were measured by qPCR. Forward and reverse primers were synthesized by Invitrogen (Thermo Fisher Scientific, Inc.; Table I). PCR amplification started with a preliminary denaturation step at $94^{\circ} \mathrm{C}$ for 2 min, followed by 45 cycles of $95^{\circ} \mathrm{C}$ for $30 \mathrm{sec}, 56^{\circ} \mathrm{C}$ for $30 \mathrm{sec}$ (annealing) and $72^{\circ} \mathrm{C}$ for $45 \mathrm{sec}$. The reaction volume was $20 \mu \mathrm{l}$, containing $50 \mathrm{ng}$ genomic cDNA, $200 \mu \mathrm{M}$ dNTPs, $200 \mu \mathrm{M}$ primers, $2.5 \mathrm{U}$ Taq DNA polymerase and 2.5 U SYBR-Green (Thermo Fisher Scientific, Inc.). Relative mRNA expression changes were calculated by the $2^{-\Delta \Delta C q}$ method (21). Results are presented as the $n$-fold change compared with $\beta$-actin.

Cell viability. Human OA chondrocytes $\left(1 \times 10^{3} /\right.$ well $)$ were seeded in 96-well plates following treatment with IL-1 $\beta(2.0 \mathrm{mg} / \mathrm{ml})$ and/or hesperidin $(2.0 \mathrm{mg} / \mathrm{ml}$; IL- $1 \beta$, IL-1 $\beta$ +hesperidin and hesperidin groups) for $24 \mathrm{~h}$ at $37^{\circ} \mathrm{C}$, and treated with $10 \mu \mathrm{l}$ MTT $(5 \mathrm{mg} / \mathrm{ml}$, Sigma-Aldrich; Merck $\mathrm{KGaA}$ ) for $3 \mathrm{~h}$ at $37^{\circ} \mathrm{C}$. Following incubation, purple formazan crystals were dissolved using isopropanol $(15 \mu \mathrm{l})$. The absorbance was recorded using a microplate reader (Multiskan FC, Thermo Fisher Scientific, Inc.) at $570 \mathrm{~nm}$. The effects of hesperidin on cell viability were determined by the percent of cell viability, calculated as the ratio between mean absorbance of three samples and mean absorbance of the IL-1 $\beta$ group.

Cell transfection. Human OA chondrocytes $\left(1 \times 10^{6} /\right.$ well, untreated cells) were cultured in six-well plates for $12 \mathrm{~h}$ at $37^{\circ} \mathrm{C}$. The NF- $\kappa \mathrm{B}$ overexpression plasmid was synthesized by cloning human NF- $\mathrm{KB}$ cDNA into plasmid pcDNA3.1 (pNF-кB; Suzhou GenePharma Co., Ltd., Suzhou, China) and the empty plasmid pcDNA3.1 served as the control (pControl). Cells were transfected pNF-кB (100 pmol) or pControl (100 pmol) using Lipofectamine 3000 reagent (Invitrogen, Thermo Fisher Scientific, Inc.) according to the manufacturer's protocol. Following $48 \mathrm{~h}$ transfection, NF- $\mathrm{kB}$-overexpressed cells were treated with hesperidin $(2.0 \mathrm{mg} / \mathrm{ml})$ for $24 \mathrm{~h}$ at $37^{\circ} \mathrm{C}$ further analysis.

Production of nitric oxide (NO) and prostaglandin E2 (PGE2). After $48 \mathrm{~h}$ transfection, NF-kB-overexpressed cells, treated with hesperidin or PBS, were used to detect NO and PGE2 production. Production of NO was assessed by measuring the nitrite content in the culture supernatant was obtained via centrifugation at $4,000 \mathrm{xg}$ at room temperature for $10 \mathrm{~min}$ following mixing of the culture with Griess reagent $(1 \%$ sulphanilamide, $0.1 \% \mathrm{~N}$-1-naphthylenediamine dihydrochloride and $2.5 \%$ phosphoric acid). The absorbance was measured at $540 \mathrm{~nm}$ following $10 \mathrm{~min}$ of incubation at $37^{\circ} \mathrm{C}$. The levels of PGE2 were analyzed using the Prostaglandin E2 Parameter Assay kit (KGE004B, Bio-Rad Laboratories, Inc., Hercules, CA, USA) according to the manufacturer's instructions.

Western blot analysis. After $48 \mathrm{~h}$ transfection, NF-kB-overexpressed human OA chondrocytes $\left(1 \times 10^{7}\right)$ treated with hesperidin or PBS were lysed using radioimmunoprecipitation lysis buffer (Thermo Fisher Scientific, Inc.). Protein concentrations were measured using a BCA protein assay kit (Thermo Fisher Scientific, Inc.). Equal amounts of proteins $(10 \mu \mathrm{g})$ were separated using $12 \%$ SDS-PAGE and transferred to a polyvinylidene fluoride membrane (Merck KGaA). Following blocking with $5 \%$ bovine serum albumin for $2 \mathrm{~h}$ at $37^{\circ} \mathrm{C}$, membranes were incubated with primary antibodies for $12 \mathrm{~h}$ at $4^{\circ} \mathrm{C}: \mathrm{IL}-1 \beta(1: 1,000 ;$ ab200478; Abcam, Cambridge, UK), IL-17A (1:1,000; ab193955; Abcam), IL-6 (1:1,000; ab7737; Abcam), IL-10 (1:1,000; ab19969; Abcam), COX-2 (1:1,000; ab15191; Abcam), PGE-2 (1:1,000; ab181249; Abcam), inducible nitric oxide synthase (iNOS; 1:1,000; ab15323; Abcam), MMP-3 (1:1,000; ab53015; Abcam), MMP-13 (1:1,000; ab39012; Abcam) and $\beta$-actin (1:2,000; ab8226; Abcam). Horseradish peroxidase-conjugated anti-rabbit IgG antibody (1:2,000; PV-6001; OriGene Technologies, Inc., Beijing, China) was incubated with the membrane for $24 \mathrm{~h}$ at $4^{\circ} \mathrm{C}$. Membranes were washed with PBS and visualized with an electrochemiluminescence western blotting detection reagent (Amersham; GE Healthcare, Chicago, IL, USA). The band intensities were analyzed by ImageJ software 1.0 (National Institutes of Health, Bethesda, MD, USA).

$N F-\kappa B$ activity. NF-kB-overexpressed with or without hesperidin human OA chondrocytes $\left(1 \times 10^{6} /\right.$ well $)$ were cultured in six-well plates for $12 \mathrm{~h}$ at $37^{\circ} \mathrm{C}$. The $3^{\prime}$-untranslated region (3'-UTR) sequence of NF- $\kappa B$ was inserted into the pGL3 control vector (Promega Corporation, Madison, WI, USA). The construct was referred to as NF-кB-3'-UTR. Cells were washed with PBS and transfected with NF-кB-3'-UTR or control (NF-кB-3'-mimic; Promega Corporation) using Lipofectamine 3000 (Thermo Fisher Scientific, Inc.) according to the manufacturer's instructions. Cells were collected using centrifugation in $4,000 \mathrm{xg}$ at room temperature for $15 \mathrm{~min}$ after $48 \mathrm{~h}$ at $37^{\circ} \mathrm{C}$ and Renilla luciferase activity was measured using the Dual-Luciferase Reporter Assay system (Promega Corporation) according to the manufacturer's protocols. Results were obtained from three independent experiments performed in duplicate and normalized to Renilla.

Statistical analysis. Data are expressed as the mean \pm standard deviation and statistical analysis was performed with SPSS 18.0 (SPSS, Inc., Chicago, IL, USA). Statistically significant differences between groups were analyzed by one-way analysis of variance followed by Tukey's honest significant difference test. $\mathrm{P}<0.01$ was considered to indicate a statistically significant difference. 
Table I. Primers for reverse transcription-quantitative polymerase chain reaction.

\begin{tabular}{lll}
\hline Gene & \multicolumn{1}{c}{ Forward (5'-3') } & \multicolumn{1}{c}{ Reverse $\left(5^{\prime}-3^{\prime}\right)$} \\
\hline IL-6 & TTCCATCCAGTTGCCTTCTTGG & TTCTCATTTCCACGATTTCCCAG \\
IL-1 $\beta$ & GGCTGCTTCCAAACCTTTGA & GAAGACACGGATTCCATGGT \\
IL-17A & ATGCACAGCCACCGCGACTT & CTTCATGACTGCCTCCAAGTAG \\
IL-10 & CAGTGCAGAAGAGTCGACTGCAAG & CGCTTGAGATCCTGAAATATA \\
iNOS & TCAAAACCGAGGTGTA & GTGGGTAAGTATGTAGTGC \\
MMP-3 & GTTGGTACATGGGCACTGAG & TATGGTGGTCGGTAATGGTG \\
MMP-13 & TCCCTCAGGAAGCTTGAACCTGAA & AAACCTAGGGTGTGGATGCCTCTT \\
-actin & TGCTTCCTGATGACGATGTAC & TCCTCGGAGACTGGTAATGG \\
\hline
\end{tabular}

IL, interleukin; COX-2, cyclooxygenase-2; iNOS, inducible nitric oxide synthase; MMP, matrix metalloproteinase.

\section{Results}

$I L-1 \beta$ stimulates inflammation in human OA chondrocytes. Anti-inflammatory effects of IL-1 $\beta$ on human OA chondrocytes were investigated in vitro. As illustrated in Fig. 1A, IL-1 $\beta$ significantly decreased the viability of human OA chondrocytes after $24 \mathrm{~h}$ compared with the control $(\mathrm{P}<0.01)$. IL-1 $\beta$ significantly increased iNOS, COX-2, MMP-3 and MMP-13 gene and protein expression in human $\mathrm{OA}$ chondrocytes compared with the PBS-treated cells $(\mathrm{P}<0.01$; Fig. $1 \mathrm{~B}$ and $\mathrm{C})$. These results indicated that IL- $1 \beta$ stimulated inflammation in human OA chondrocytes.

Hesperidin attenuates inflammation in IL-1 $\beta$-stimulated human OA chondrocytes. Effects of hesperidin on inflammation in IL-1 $\beta$-stimulated human OA chondrocytes were investigated in vitro. Hesperidin significantly increased the viability of human OA chondrocytes compared with the PBS-treated group $(\mathrm{P}<0.01$; Fig. $2 \mathrm{~A})$. Hesperidin treatment significantly decreased IL-1 $\beta$ and IL-17A gene and protein expression $(\mathrm{P}<0.01$; Fig. $2 \mathrm{~B}$ and $\mathrm{C})$ and significantly increased IL-6 and IL-10 gene and protein expression in IL-1 $\beta$-stimulated human OA chondrocytes compared with the PBS-treated cells $(\mathrm{P}<0.01$; Fig. 2D and E). These results indicated that hesperidin may attenuate inflammation in IL-1 $\beta$-stimulated human OA chondrocytes.

Hesperidin inhibits $I L-1 \beta$-induced MMP-3 and MMP-13 release in human $O A$ chondrocytes. Hesperidin effects on NO, PGE2, iNOS and COX-2 expression in IL-1 $\beta$-stimulated human $\mathrm{OA}$ chondrocytes were further investigated. It was revealed that hesperidin significantly decreased NO and PGE2 production $(\mathrm{P}<0.01$; Fig. $3 \mathrm{~A}$ and $\mathrm{B})$ and significantly inhibited iNOS and COX-2 gene and protein expression in IL-1 $\beta$-stimulated OA chondrocytes compared with the PBS-treated cells $(\mathrm{P}<0.01$; Fig. 3C and D). Hesperidin further significantly inhibited IL-1 $\beta$-induced MMP-3 and MMP-13 release in human $\mathrm{OA}$ chondrocytes compared with the control (PBS-treated cells; $\mathrm{P}<0.01$; Fig. 3E and F). These results suggested that hesperidin may inhibit the IL-1 $\beta$-induced NO and MMP signaling pathways in human OA chondrocytes.
Hesperidin affects IL-1 $\beta$-stimulated inflammation by inhibiting $N F-\kappa B$ activity in human OA chondrocytes. The potential mechanism mediated by hesperidin was analyzed in human OA chondrocytes. It was observed that hesperidin significantly decreased the activity of $\mathrm{NF}-\kappa \mathrm{B}$ in IL-1 $\beta$-stimulated human OA chondrocytes compared with the PBS-treated cells $(\mathrm{P}<0.01$; Fig. 4A). Additionally, NF- $\kappa$ BIR treatment significantly decreased iNOS, COX-2, MMP-3 and MMP-13 gene and protein expression in IL-1 $\beta$-stimulated human OA chondrocytes compared with the PBS-treated cells $(\mathrm{P}<0.01$; Fig. $4 \mathrm{~B}$ and $\mathrm{C})$. NF- $\kappa \mathrm{B}$ overexpression, induced by transfection with $\mathrm{pNF}-\kappa \mathrm{B}$, significantly increased iNOS, COX-2, MMP-3 and MMP-13 gene and protein expression in IL-1 $\beta$-stimulated human OA chondrocytes compared with the pControl ( $\mathrm{P}<0.01$; Fig. 4D and $\mathrm{E})$. The results also demonstrated that $\mathrm{NF}-\kappa \mathrm{B}$ overexpression reversed hesperidin-inhibited iNOS, COX-2, MMP-3 and MMP-13 gene and protein expression in IL- $1 \beta$-stimulated human OA chondrocytes compared with the PControl group (Fig. 4F and G). These results indicated that hesperidin inhibited IL-1 $\beta$-stimulated inflammation by the inhibiting $\mathrm{NF}-\kappa \mathrm{B}$ activity in human OA chondrocytes.

\section{Discussion}

A previous study revealed that hesperidin attenuates inflammation and oxidative damage in the pleural exudates and the liver of a rat model of pleurisy (22). However, the role of hesperidin in human OA chondrocytes is yet to be elucidated. In the current study, the role of hesperidin in IL-1 $\beta$-stimulated human OA chondrocytes was analyzed. The findings revealed that hesperidin significantly decreased IL-1 $\beta$ and IL-17A gene and protein expression, while it increased IL- 6 and IL-10 gene and protein expression in IL-1 $\beta$-stimulated human OA chondrocytes compared with the PBS group. The current study observed that hesperidin inhibited IL-1 $\beta$-stimulated inflammation in human OA chondrocytes.

Currently, anti-IL-1 $\beta$ therapy is an efficient treatment method for patients with OA (23). Liu et al (24) indicated that IL-17 serves a crucial role in the pathogenesis of OA and is closely associated with pain, which suggests that blocking 
A

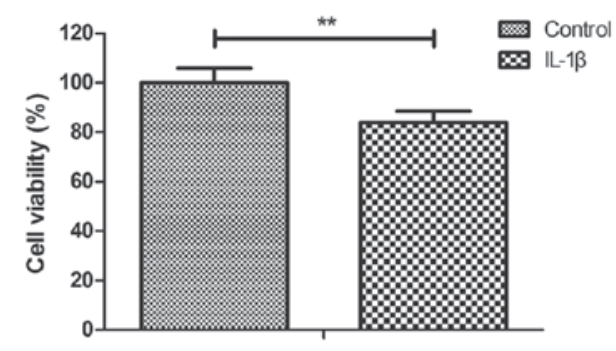

C

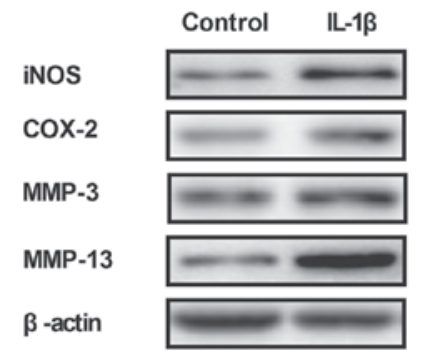

B
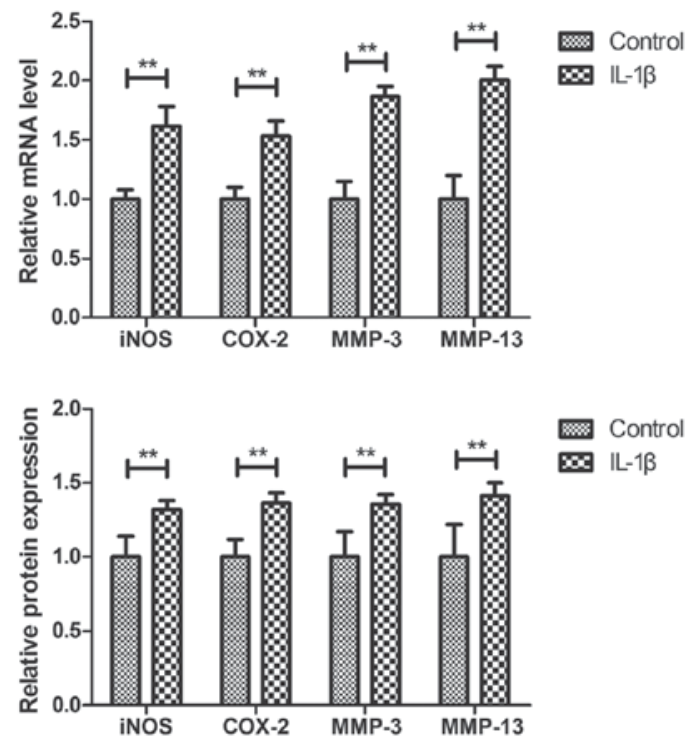

Figure 1. Effects of IL-1 $\beta$ on viability and inflammatory cytokine expression in human OA chondrocytes. (A) Effects of IL-1 $\beta$ on viability of human OA chondrocytes in vitro. IL-1 $\beta$ increased iNOS, COX-2, MMP-3 and MMP-13 (B) gene and (C) protein expression in human OA chondrocytes. ${ }^{* *} \mathrm{P}<0.01$. OA, osteoarthritis; IL, interleukin; iNOS, inducible nitric oxide synthase; COX-2, cyclooxygenase-2; MMP, matrix metalloproteinase. Control group, PBS-treated cells.

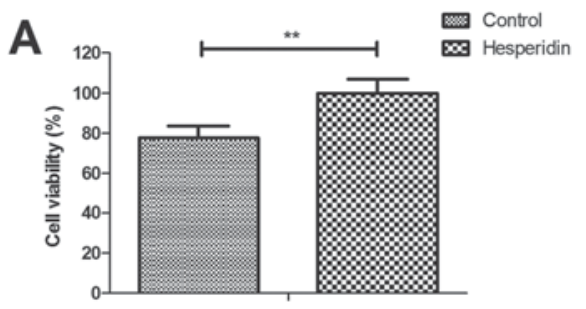

B

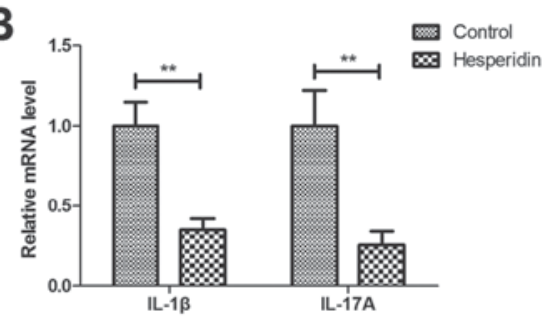

D

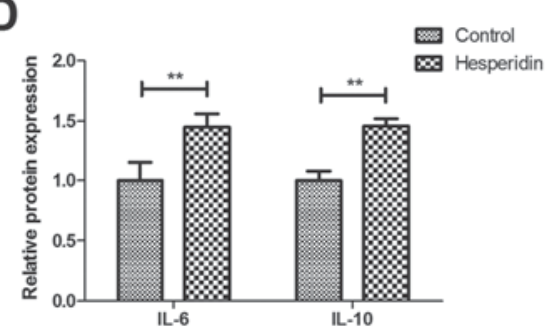

C
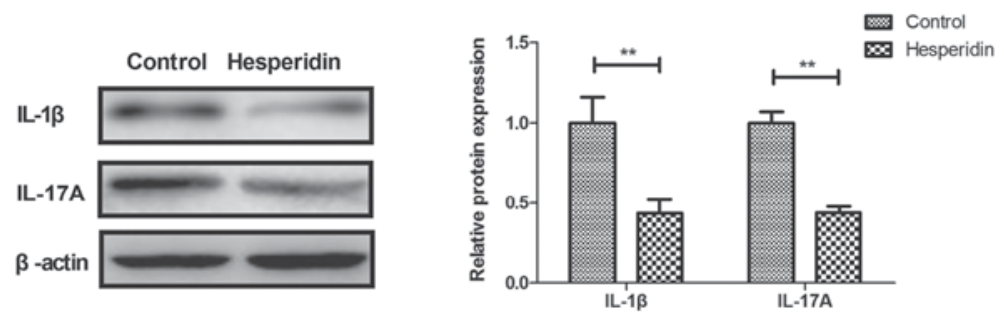

E
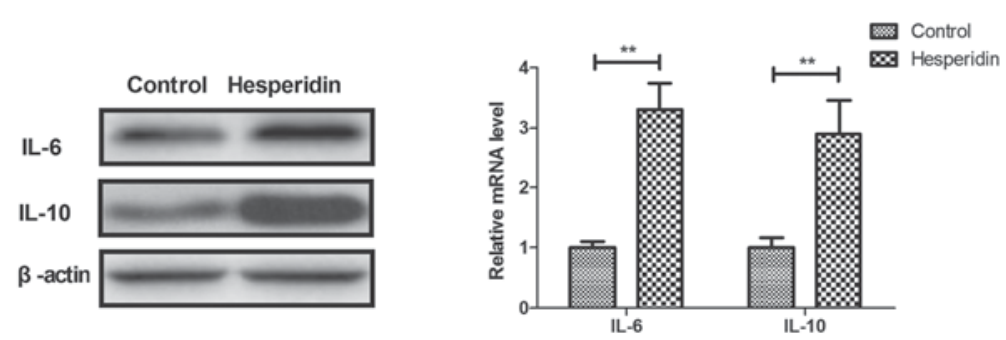

Figure 2. Hesperidin regulates inflammation in IL-1ß-stimulated human OA chondrocytes. (A) Hesperidin increases the viability of human OA chondrocytes in vitro. Effects of hesperidin on IL-1 $\beta$ and IL-17A (B) gene and (C) protein expression in IL-1 $\beta$-stimulated human OA chondrocytes. Effects of hesperidin treatment on IL-6 and IL-10 (D) gene and (E) protein expression in IL-1 $\beta$-stimulated human OA chondrocytes. ${ }^{* *} \mathrm{P}<0.01$. OA, osteoarthritis; IL, interleukin. Control group, PBS-treated cells.

the IL-17 signaling pathway may contribute to the treatment of OA. It was reported in the current study that hesperidin decreased IL-1 $\beta$ and IL-17A and increased IL-6 and IL-10 gene and protein expression in human OA chondrocytes, which has previously been indicated to be beneficial in OA therapy (24). 
A

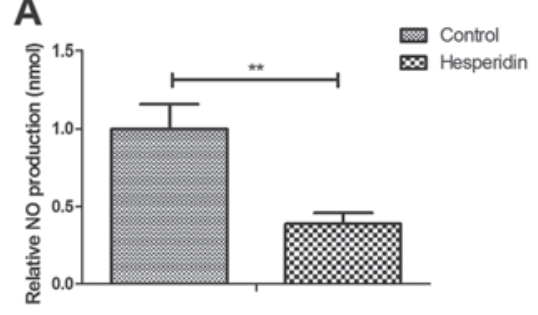

D

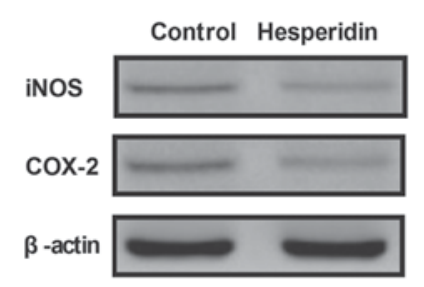

E

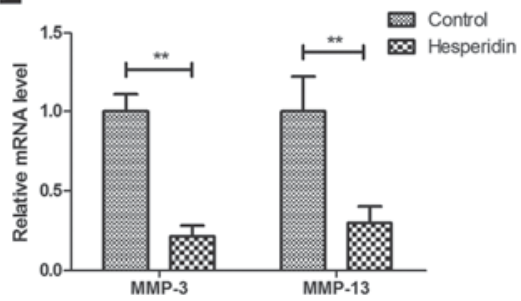

B
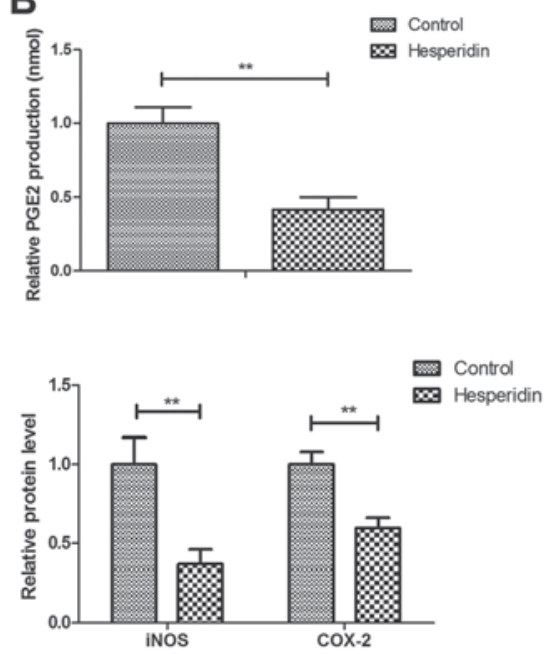

$\mathbf{F}$
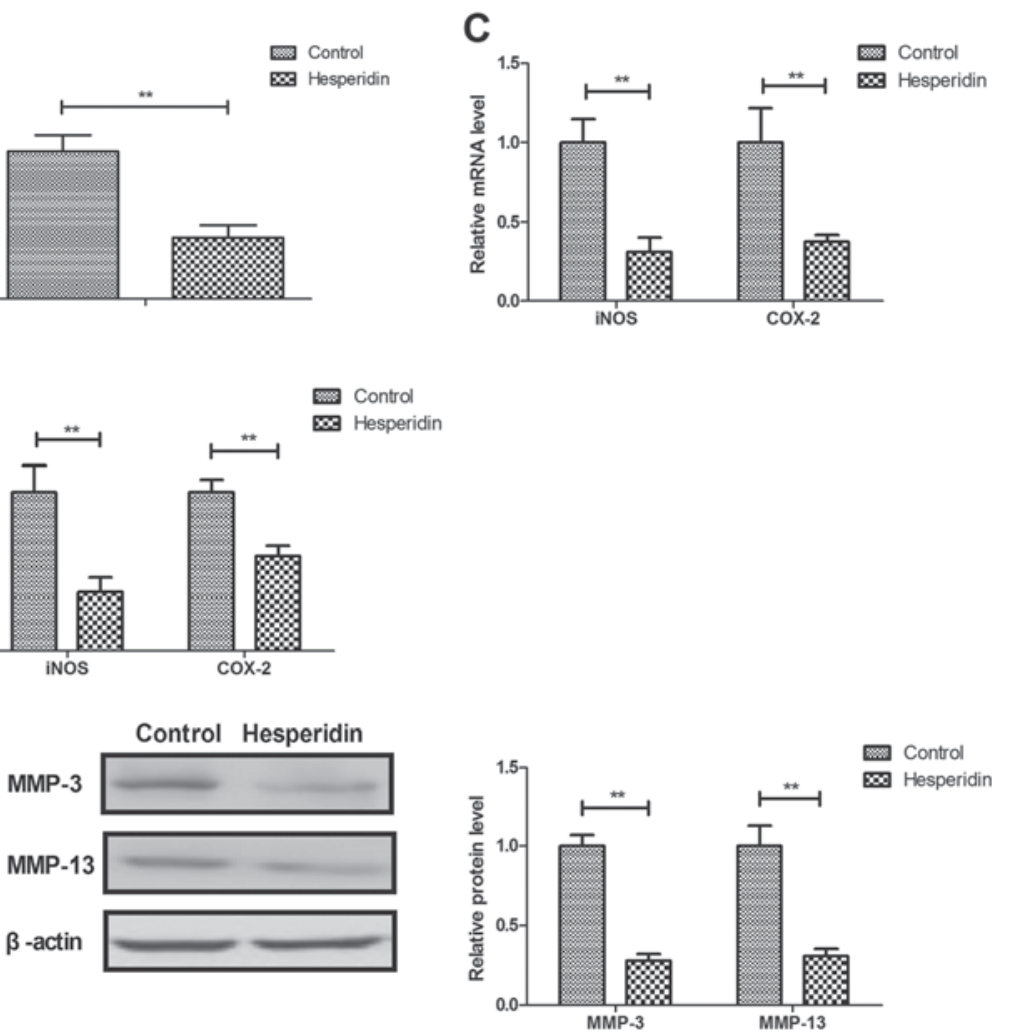

Figure 3. Effects of hesperidin on IL-1 $\beta$-induced inflammation in human OA chondrocytes. Effects of hesperidin on (A) NO and (B) PGE2 production in IL-1 $\beta$-stimulated OA chondrocytes. Effects of hesperidin on iNOS and COX-2 (C) gene and (D) protein expression in IL-1 $\beta$-stimulated human OA chondrocytes. Effects of hesperidin on IL-1 $\beta$-induced MMP-3 and MMP-13 (E) gene and (F) protein expression in human OA chondrocytes. ${ }^{* *} \mathrm{P}<0.01$ vs. control. OA, osteoarthritis; IL, interleukin; NO, nitric oxide; PGE2, prostaglandin E2; iNOS, inducible NO synthase; COX-2, cyclooxygenase-2; MMP, matrix metalloproteinase. Control group, PBS-treated cells.

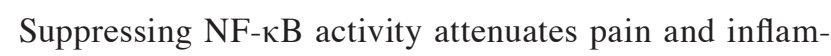
mation in rats with osteoarthritis (25). Hesperidin has been reported to suppress oxidative stress and inflammation via activation of the protein kinase $\mathrm{B} /$ nuclear factor erythroid 2-related factor 2 signaling pathway, inhibition of the receptor for advanced glycation end products/NF- $\mathrm{\kappa B}$ signaling pathway and further confers neuroprotection (26). In the current study, it was revealed that hesperidin significantly inhibited NF- $\mathrm{KB}$ activity in IL-1 $\beta$-stimulated human OA chondrocytes.

Suppression of PGE2 and MMP expression was indicated to inhibit cartilage degradation by pomegranate fruit extracts in a model of posttraumatic osteoarthritis (27). Another study reported that patients with OA exhibited significantly higher COX-2 levels compared with the healthy control group (28). In the current study, findings demonstrated that hesperidin significantly decreased PGE2 and COX-2 expression in IL-1 $\beta$-stimulated human OA chondrocytes compared with PBS-treated cells. A previous study has demonstrated that demethylation of an NF- $\mathrm{KB}$ enhancer element induced iNOS production in OA and is associated with an altered chondrocyte cell cycle (29). In the current study, results revealed that hesperidin inhibited iNOS and COX-2 gene and protein expression in IL-1 $\beta$-stimulated human OA chondrocytes compared with PBS-treated cells. Findings suggested that hesperidin regulated iNOS and COX-2 expression via the inhibition of NF- $\kappa B$ activity in IL-1 $\beta$-stimulated human OA chondrocytes compared with PBS-treated cells.
In conclusion, the findings of the current study suggest that hesperidin downregulated inflammation in IL-1 $\beta$-stimulated human OA chondrocytes and highlight the potential application of hesperidin for OA therapy based on decreasing NF- $\mathrm{KB}$ activity. The current study suggests that hesperidin may be a potential agent for the inhibition of inflammation in the treatment of OA.

\section{Acknowledgements}

Not applicable.

\section{Funding}

The current study was supported by the Natural Science Foundation of Guangdong Province (grant no. 2015A030310248).

\section{Availability of data and materials}

The datasets used and/or analyzed during the current study are available from the corresponding author on reasonable request.

\section{Authors' contributions}

ZF designed the experiments. ZC and QX performed the experiments. HL and SX acquired and interpreted all data 
A

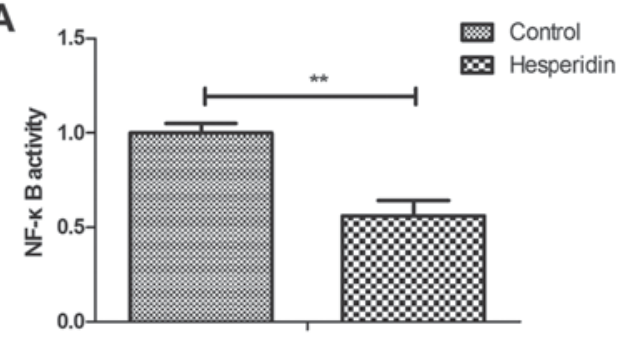

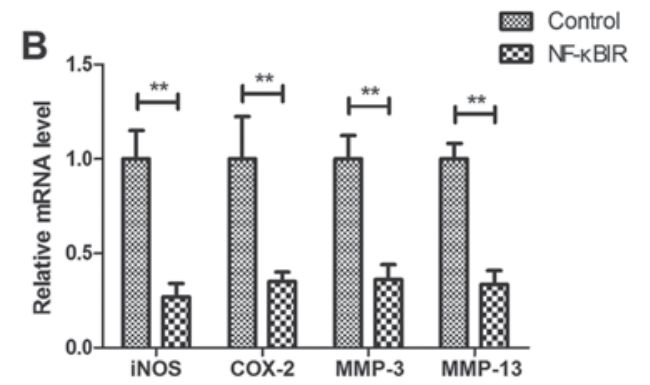

D

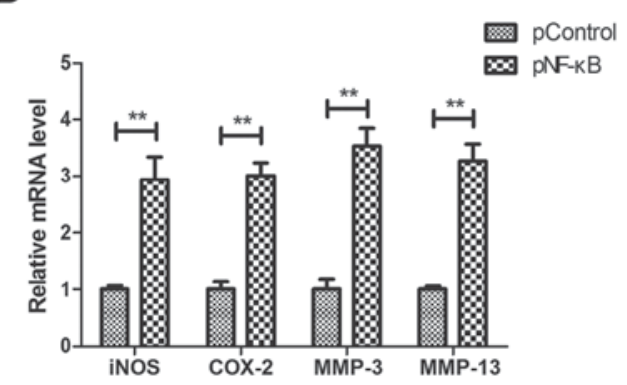

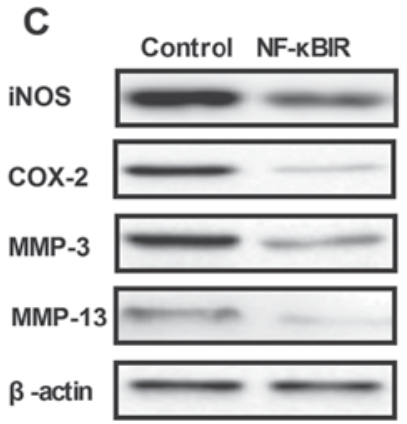

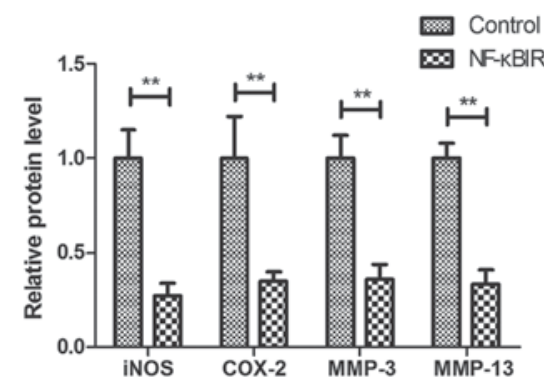

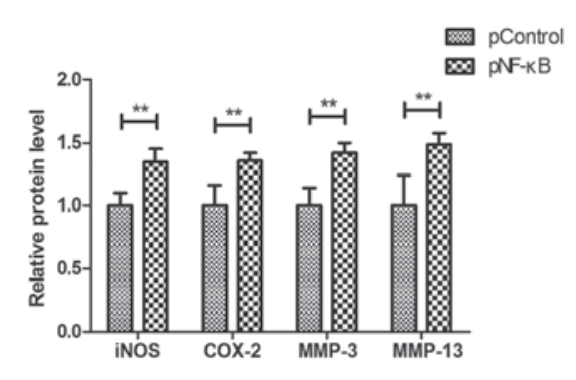

$\mathbf{F}$
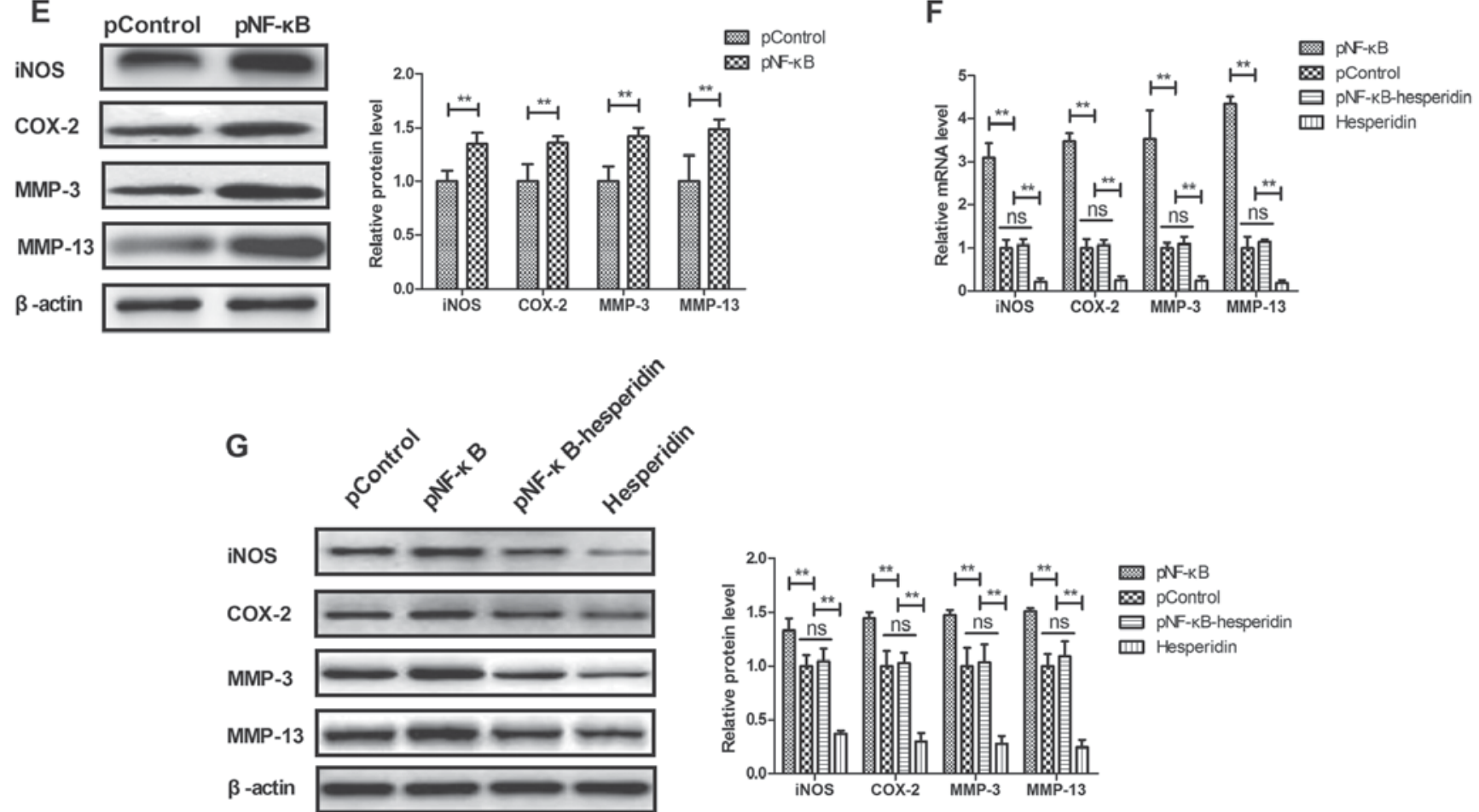

Figure 4. Hesperidin inhibits IL-1 $\beta$-stimulated inflammation by reducing NF- $\kappa B$ activity in human OA chondrocytes. (A) Hesperidin decreases the activation

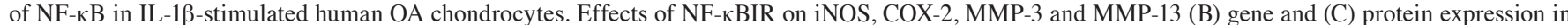
IL-1 $\beta$-stimulated human OA chondrocytes. Effects of pNF- $\mathrm{kB}$ on iNOS, COX-2, MMP-3 and MMP-13 (D) gene and (E) protein expression in IL-1 $\beta$-stimulated

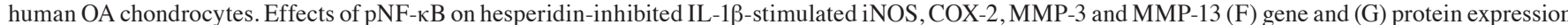

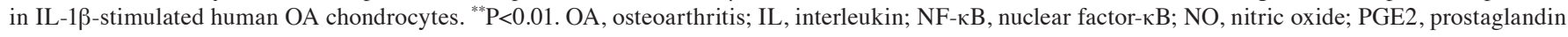

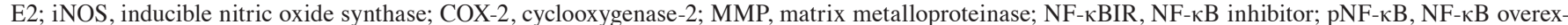
pression; pControl, empty pcDNA3.1.

in the present study. All authors read and approved the final manuscript.

\section{Ethics approval and consent to participate}

The current study was approved by the Ethical Committee of Jiangmen Central Hospital, Jiangmen (Jiangmen, China).

\section{Patient consent for publication}

Not applicable.

\section{Competing interests}

The authors declare that they have no competing interests. 


\section{References}

1. Onuora S: Osteoarthritis: Molecular imaging detects activated macrophages. Nat Rev Rheumatol 12: 313, 2016

2. Meheux CJ, McCulloch PC, Lintner DM, Varner KE and Harris JD: Efficacy of intra-articular platelet-rich plasma injections in knee osteoarthritis: A systematic review. Arthroscopy 32: 495-505, 2016

3. Monticone M, Frizziero A, Rovere G, Vittadini F, Uliano D, LA Bruna S, Gatto R, Nava C, Leggero V and Masiero S: Hyaluronic acid intra-articular injection and exercise therapy: Effects on pain and disability in subjects affected by lower limb joints osteoarthritis. A systematic review by the italian society of physical and rehabilitation medicine (SIMFER). Eur J Phys Rehabil Med 52: 389-399, 2016.

4. Hammond A, Jones V and Prior Y: The effects of compression gloves on hand symptoms and hand function in rheumatoid arthritis and hand osteoarthritis: A systematic review. Clin Rehabil 30: 213-224, 2016.

5. Tang H, He S, Zhang X, Luo S, Zhang B, Duan X, Zhang Z, Wang W, Wang Y and Sun Y: A network pharmacology approach to uncover the pharmacological mechanism of XuanHuSuo powder on osteoarthritis. Evid Based Complement Alternat Med 2016: 3246946, 2016.

6. Roemer FW, Kwoh CK, Hannon MJ, Hunter DJ, Eckstein F, Grago J, Boudreau RM, Englund M and Guermazi A: Partial meniscectomy is associated with increased risk of incident radiographic osteoarthritis and worsening cartilage damage in the following year. Eur Radiol 27: 404-413, 2017.

7. Malfait AM: Osteoarthritis year in review 2015: Biology. Osteoarthritis Cartilage 24: 21-26, 2016.

8. Maleki M, Arazpour M, Joghtaei M, Hutchins SW, Aboutorabi A and Pouyan A: The effect of knee orthoses on gait parameters in medial knee compartment osteoarthritis: A literature review. Prosthet Orthot Int 40: 193-201, 2016.

9. Li Y, Su Y, Chen S, Zhang Y, Zhang Z, Liu C, Lu M, Liu F, Li S, $\mathrm{He} Z$, et al: The effects of resistance exercise in patients with knee osteoarthritis: A systematic review and meta-analysis. Clin Rehabil 30: 947-959, 2016.

10. La Torre F, Nicolai AP and Otti M: Hemorrhoids and conservative treatment. Review of the literature on the use of diosmin and micronized hesperidin. Minerva Chir 54: 909-916, 1999 (In Italian).

11. Roohbakhsh A, Parhiz H, Soltani F, Rezaee R and Iranshahi M: Neuropharmacological properties and pharmacokinetics of the citrus flavonoids hesperidin and hesperetin-a mini-review. Life Sci 113: 1-6, 2014.

12. Antunes MS, Goes AT, Boeira SP, Prigol M and Jesse CR: Protective effect of hesperidin in a model of Parkinson's disease induced by 6 -hydroxydopamine in aged mice. Nutrition 30 $1415-1422,2014$

13. Wang D, Liu L, Zhu X, Wu W and Wang Y: Hesperidin alleviates cognitive impairment, mitochondrial dysfunction and oxidative stress in a mouse model of Alzheimer's disease. Cell Mol Neurobiol 34: 1209-1221, 2014.

14. Kumar A, Chaudhary T and Mishra J: Minocycline modulates neuroprotective effect of hesperidin against quinolinic acid induced Huntington's disease like symptoms in rats: Behavioral, biochemical, cellular and histological evidences. Eur J Pharmacol 720: 16-28, 2013.
15. Li G, Chen MJ, Wang C, Nie H, Huang WJ, Yuan TD, Sun T, Shu KG, Wang CF, Gong Q and Tang SQ: Protective effects of hesperidin on concanavalin A-induced hepatic injury in mice. Int Immunopharmacol 21: 406-411, 2014.

16. Poetini MR, Araujo SM, Trindade de Paula M, Bortolotto VC, Meichtry LB, Polet de Almeida F, Jesse CR, Kunz SN and Prigol M: Hesperidin attenuates iron-induced oxidative damage and dopamine depletion in Drosophila melanogaster model of Parkinson's disease. Chem Biol Interact 279: 177-186, 2018.

17. Ahmad ST, Arjumand W, Nafees S, Seth A, Ali N, Rashid S and Sultana S: Hesperidin alleviates acetaminophen induced toxicity in Wistar rats by abrogation of oxidative stress, apoptosis and inflammation. Toxicol Lett 208: 149-161, 2012.

18. Hochberg MC: Treatment of rheumatoid arthritis and osteoarthritis with COX-2-selective inhibitors: A managed care perspective. Am J Manag Care 8 (17 Suppl): S502-S517, 2002.

19. Tchetverikov I, Lohmander LS, Verzijl N, Huizinga TW, TeKoppele JM, Hanemaaijer R and DeGroot J: MMP protein and activity levels in synovial fluid from patients with joint injury, inflammatory arthritis, and osteoarthritis. Ann Rheum Dis 64: 694-698, 2005.

20. Xu L, Sun C, Zhang S, Xu X, Zhai L, Wang Y, Wang S, Liu Z, Cheng $\mathrm{H}$, Xiao M, et al: Sam68 promotes NF- $\mathrm{kB}$ activation and apoptosis signaling in articular chondrocytes during osteoarthritis. Inflamm Res 64: 895-902, 2015.

21. Livak KJ and Schmittgen TD: Analysis of relative gene expression data using real-time quantitative PCR and the 2(-Delta Delta C(T)) method. Methods 25: 402-408, 2001.

22. Adefegha SA, Rosa Leal DB, Olabiyi AA, Oboh G and Castilhos LG: Hesperidin attenuates inflammation and oxidative damage in pleural exudates and liver of rat model of pleurisy. Redox Rep 22: 563-571, 2017.

23. Chevalier X, Conrozier T and Richette P: Desperately looking for the right target in osteoarthritis: The anti-IL-1 strategy. Arthritis Res Ther 13: 124, 2011.

24. Liu Y, Peng H, Meng Z and Wei M: Correlation of IL-17 level in synovia and severity of knee osteoarthritis. Med Sci Monit 21: 1732-1736, 2015.

25. Dai T, Shi K, Chen G, Shen Y and Pan T: Malvidin attenuates pain and inflammation in rats with osteoarthritis by suppressing $\mathrm{NF}-\kappa \mathrm{B}$ signaling pathway. Inflamm Res 66: 1075-1084, 2017.

26. Hong Y and An Z: Hesperidin attenuates learning and memory deficits in APP/PS1 mice through activation of Akt/Nrf2 signaling and inhibition of RAGE/NF- $\mathrm{KB}$ signaling. Arch Pharm Res 41: 655-663, 2018

27. Akhtar N, Khan NM, Ashruf OS and Haqqi TM: Inhibition of cartilage degradation and suppression of PGE2 and MMPs expression by pomegranate fruit extract in a model of posttraumatic osteoarthritis. Nutrition 33: 1-13, 2017.

28. Fan HW, Liu GY, Zhao CF, Li XF and Yang XY: Differential expression of COX-2 in osteoarthritis and rheumatoid arthritis. Genet Mol Res 14: 12872-12879, 2015.

29. de Andrés MC, Takahashi A and Oreffo RO: Demethylation of an NF- $\kappa \mathrm{B}$ enhancer element orchestrates iNOS induction in osteoarthritis and is associated with altered chondrocyte cell cycle. Osteoarthritis Cartilage 24: 1951-1960, 2016. 